\title{
Heat capacity and thermal conductivity of multiferroics $\mathrm{Bi}_{1-\mathrm{x}} \mathrm{Pr}_{\mathrm{x}} \mathrm{FeO}_{3}$
}

\author{
S. N. Kallaeva, Z. M. Omarov', R.G. Mitarov ${ }^{\text {b }}$, S. A. Sadykov', S. V. Khasbulatov', \\ L. A. Reznichenko ${ }^{d}, K$. Bormanis ${ }^{\mathrm{e}}$, and M. Kundzinsh ${ }^{\mathrm{e}}$ \\ ${ }^{a}$ Dagestan Science Centre, Institute of Physics, RAS, Makhachkala, Russia; ${ }^{b}$ Dagestan State Technical \\ University, Makhachkala, Russia; 'Physical Department, Dagestan State University, Makhachkala, Russia;

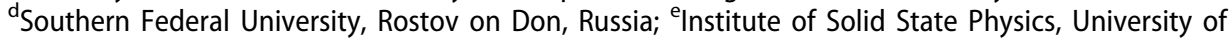 \\ Latvia, Riga, Latvia
}

\begin{abstract}
The heat capacity and thermal conductivity of multiferroics $\mathrm{Bi}_{1-x} \mathrm{Pr}_{x} \mathrm{FeO}_{3}(0 \leq x \leq 0.50)$ has been studied in the temperature range of $130-800 \mathrm{~K}$. A slight substitution of praseodymium for bismuth is found to lead to a noticeable shift of the antiferromagnetic phase transition temperature whilst the heat capacity increases. The temperature dependences of the heat capacity and thermal conductivity exhibit additional anomalies during phase transitions. The experimental results suggest that the excess heat capacity can be attributed to the Schottky effect for three-level states. The basic mechanisms of the heat transfer of phonons are highlighted and the dependence of the mean free path on temperature is determined.
\end{abstract}

\section{ARTICLE HISTORY}

Received 2 October 2018

Accepted 8 February 2019

\section{KEYWORDS}

Multiferroics; heat capacity; thermal conductivity

\section{Introduction}

In recent years there has been a surge of researchers' interest in crystal structure and physical properties of multiferroics, i.e. materials characterized by coexistence of magnetic and ferroelectric orderings. Researchers' interest in such materials has been prompted by the potentials of practical applications. Multiferroics can be used to develop magnetic field sensors, data recorder/reader, spinelectronics and microwave ovens and other devices. These promising materials include bismuth ferrite $\mathrm{BiFeO}_{3}$, which undergoes the ferroelectric (at $T_{\mathrm{c}} \sim 1083 \mathrm{~K}$ ) and the anti-ferromagnetic (at $T_{\mathrm{N}} \sim$ $643 \mathrm{~K}$ ) phase transitions [1]. Bismuth ferrite has space group $R 3 c$ at room temperature. The crystal structure is characterized by the presence of rhombohedrally distorted perovskite cells approximating the cubic shape. At temperatures below the Néel point $T_{\mathrm{N}}$, bismuth ferrite exhibits a complex spatially-modulated cycloid-type magnetic structure that does not allow the existence of ferromagnetic properties [2]. A necessary condition for the appearance of the magnetoelectric effect is destruction of its spatially-modulated spin structure that can be obtained by doping the bismuth ferrite with rareearth elements.

Results of studies of ceramic $\mathrm{BiFeO}_{3}$ samples modified with rare-earth elements demonstrate some difference of opinions concerning the sequence of structural phase 
transitions and the temperature ranges of the existence of different phases with substitutions of various rare-earth elements and with their increased concentration.

The temperature dependence of crystal structure of solid solutions $\mathrm{Bi}_{1-x} \mathrm{Pr}_{x} \mathrm{FeO}_{3}$ is different from the structural changes observed in the $\mathrm{BiFeO}_{3}$ compositions with other rare-earth ions substitution $[3,4]$. It was demonstrated $[5,6]$ that at increasing temperatures the $\mathrm{Bi}_{1-x} \mathrm{Pr}_{x} \mathrm{FeO}_{3}$ compositions have a tendency to form a two-phase and threephase structural states in a certain temperature range, in which the rhombohedral and the orthorhombic phases should coexist. The results encourage further studies of bismuth ferrite based multiferroics doped by rare-earth elements. In particular, the calorimetric measurements over a wide temperature range make it possible to detect anomalies of the heat capacity, yielding important information on the nature of the involved physical phenomena. It is worthwhile to mention, that the heat capacity and thermal conductivity of the $\mathrm{Bi}_{1-x} \mathrm{Pr}_{x} \mathrm{FeO}_{3}$ system has not yet been a subject of extensive studies. Earlier in our work [7] the results of studies of the heat capacity of $\mathrm{Bi}_{1-x} \mathrm{Pr}_{x} \mathrm{FeO}_{3}$ of some compositions with concentrations $0 \leq x \leq 0.20$ were presented. This study investigates the heat capacity and thermal conductivity of multiferroics $\mathrm{Bi}_{1-x} \mathrm{Pr}_{x} \mathrm{FeO}_{3}$ with concentrations $0 \leq x \leq 0.50$ over a wide temperature range $130-800 \mathrm{~K}$.

\section{Experimental}

Ceramic samples of $\mathrm{Bi}_{1-x} \mathrm{Pr}_{x} \mathrm{FeO}_{3}$ solid solutions with $x=0,0.05,0.10,0.15,0.20,0.30$, 0.40 and 0.50 were prepared the popular ceramic technology involving solid-phase synthesis with subsequent sintering in air without additional pressure. The synthesis was performed using high-purity oxides for two stages with intermediate milling and granulating the powders. The synthesis conditions were as follows: the first stage sintering calcination was at temperature $T_{1}=800^{\circ} \mathrm{C}\left(\tau_{1}=10 \mathrm{~h}\right)$, and the second stage sintering was performed at $T_{2}=800-850^{\circ} \mathrm{C}\left(\tau_{2}=5 \mathrm{~h}\right)$. A plasticizer with subsequent granulation was introduced into the powder to give it the properties required for pressing. The optimal sintering temperature was chosen in the range $900 \leq T_{\text {ann }} \leq 950{ }^{\circ} \mathrm{C}$. The $\mathrm{X}$-ray powder diffraction analyses were performed using a DRON-3 diffractometer with $\mathrm{Fe} K_{\alpha}$ and $\mathrm{Cu} K_{\alpha}$ radiations. Thus prepared solid solutions had fairly high relative (89-94\%) densities, which corresponds to the density limits achieved in the common ceramic technology (90-95\%) that indicated sufficiently high quality of the ceramics.

The heat capacity was measured using the NETZSCH DSC 204 F1Phoenix® differential scanning calorimeter. The samples were in the form of $1 \mathrm{~mm}$ thick plate with $4 \mathrm{~mm}$ diameter. The temperature was varied with step $5 \mathrm{~K} / \mathrm{min}$. The heat capacity was measured with accuracy $3 \%$.

Studies of thermal diffusivity and thermal conductivity were carried out by laser flash on the LFA-457 MicroFlash unit from NETZSCH. Samples used were $12.5 \mathrm{~mm}$ in diameter and $1 \mathrm{~mm}$ in thickness. Thermal conductivity was derived by the formula $\lambda=\eta \cdot \mathrm{Cp} \cdot \rho$ (where $\eta$ is the thermal diffusivity, $\rho$ is the density of sample, Cp heat capacity). 


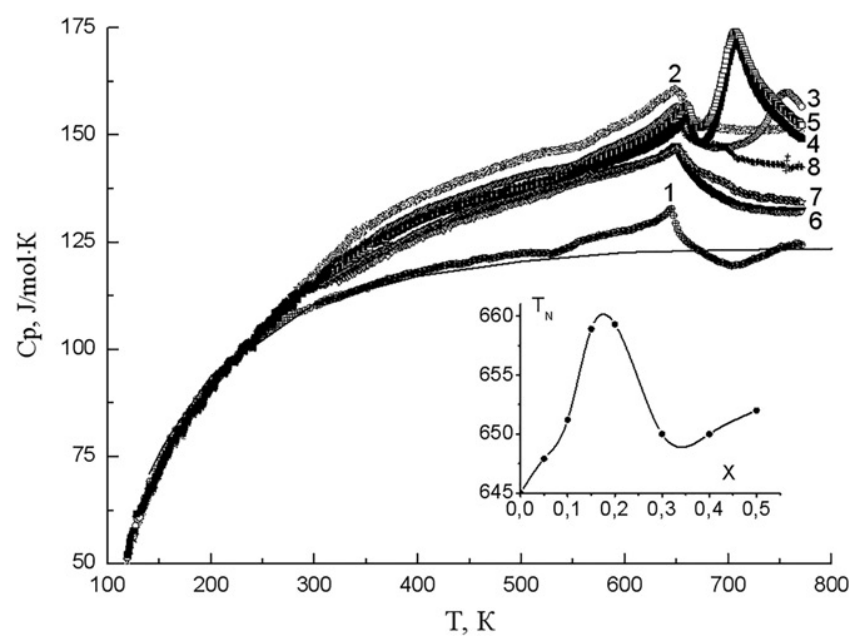

Figure 1. Temperature dependence of heat capacity of $\mathrm{Bi}_{1-x} \mathrm{Pr}_{x} \mathrm{FeO}_{3}$ with $x=0$ (1), 0.05 (2), 0.10 (3), $0.15(4), 0.20$ (5), 0.30 (6), 0.40 (7) and 0.50 (8); solid line is shown the result of the approximation of the phonon heat capacity by the Debye function. The inset: $T_{N^{-}} X$ phase diagram of the $\mathrm{Bi}_{1-x} \mathrm{Pr}_{x} \mathrm{FeO}_{3}$ system.

\section{Results and discussion}

The X-ray phase analysis data show that the impurity-free solid solutions were formed exclusively at the $\operatorname{Pr}$ concentration $x \geq 0.12$; at lower $x$ the samples contained low concentration of the $\mathrm{Bi}_{25} \mathrm{FeO}_{40}$ and $\mathrm{Bi}_{2} \mathrm{Fe}_{4} \mathrm{O}_{9}$ compounds. The analysis of the diffraction reflections revealed several concentration ranges with different phase compositions. In the range $0 \leq x<0.10$, the rhombohedral $(R 3 c)$ phase (inherent to $\mathrm{BiFeO}_{3}$ ) was found to exist. Three phases coexisted at $0.10<x \leq 0.20$ : one rhombohedral $R 3 c$ and two orthorhombic $P_{1}$-type $\mathrm{PbZrO}_{3}$ and $P_{2}$-type $\mathrm{GdFeO}_{3}$ (the former phase was dominant). In the range of $0.20<x \leq 0.30$, phase $R 3 c$ disappeared, but two phases $P_{1}$ and $P_{2}$ coexisted. A similar situation was observed during experiments with solid solutions $\mathrm{Bi}_{1-x} \mathrm{La}_{x} \mathrm{FeO}_{3}$ [4] and $\mathrm{Bi}_{1-x} \mathrm{Pr}_{x} \mathrm{FeO}_{3}[5,6,8]$.

Figure 1 shows the heat capacity $C_{\mathrm{p}}$ of $\mathrm{Bi}_{1-x} \mathrm{Pr}_{x} \mathrm{FeO}_{3}(x=0,0.05,0.10,0.15,0.20$, $0.30,0.40$ and 0.50 ) in the temperature range of $130-800 \mathrm{~K}$. It is apparent (Figure 1) that, the temperature dependences of the heat capacity for all compositions exhibit anomalies near the antiferromagnetic phase transition temperature $T_{\mathrm{N}}$, and $C_{\mathrm{p}}$ temperature shifts to higher values as $x$ increases $(0<x \leq 0.20)$, whilst $\mathrm{T}_{\mathrm{N}}$ tends to lower temperatures in compositions with $x>0.2$. When $\mathrm{BiFeO}_{3}$ is substituted by praseodymium the heat capacity increases in a wide temperature ranges above $T \geq 240 \mathrm{~K}$. The inset in Figure 1 presents the $T_{\mathrm{N}^{-}} x$ phase diagram of the $\mathrm{Bi}_{1-x} \mathrm{Pr}_{x} \mathrm{FeO}_{3}$ system plotted basing on results of the heat capacity measurements. The heat capacity $v s$ temperature curves for $x=0.10, x=0.15$ and 0.20 exhibit the second anomalies, typical for phase transitions, at temperatures higher than $T_{\mathrm{N}}: T \approx 755 \mathrm{~K}$ and $\approx 710 \mathrm{~K}$, respectively. According to the $\mathrm{X}$ ray diffraction studies of $\mathrm{Bi}_{1-x} \mathrm{Pr}_{x} \mathrm{FeO}_{3}[5,6]$, we can suppose that the heat capacity anomalies at $T \approx 710 \mathrm{~K}$ (Figure 1a) for the compositions with $x=0.15$ and 0.20 could be attributed to the phase transition between the antipolar and nonpolar orthorhombic Pnam and Pnma structures, while the anomalous behavior of the heat capacity for 


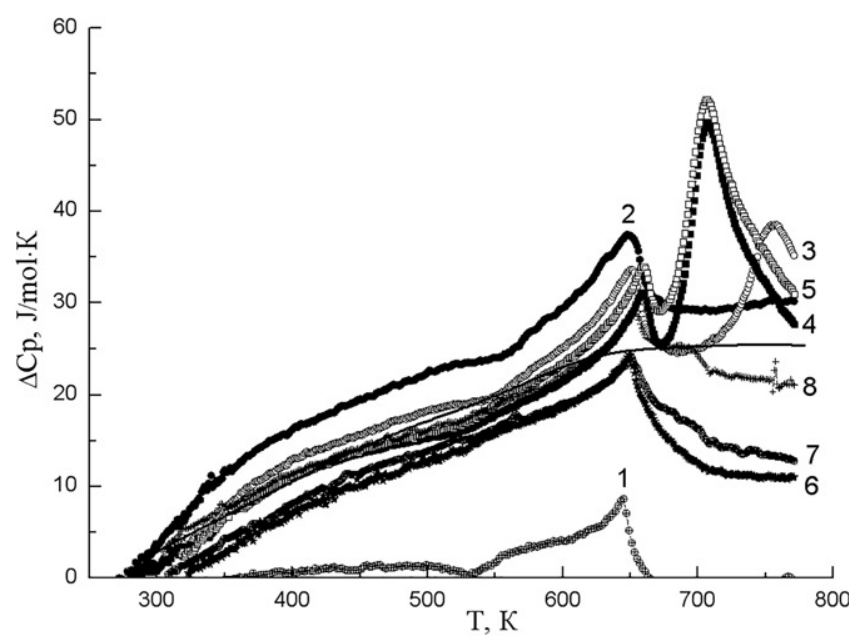

Figure 2. Temperature dependence of the anomalous component of heat capacity of $\mathrm{Bi}_{1-x} \mathrm{Pr}_{x} \mathrm{FeO}_{3}$ for $x=0$ (1), 0.05 (2), 0.10 (3), $0.15(4), 0.20$ (5), 0.30 (6), 0.40 (7) and 0.50 (8); solid line is shown the result of the approximation by Eq. (1).

$x=0.10$ at $T \approx 755 \mathrm{~K}$ is related to the structural phase transition between rhombohedral R3c and orthorhombic Pnma structures.

The experimental values of heat capacity over a wide temperature range should be analyzed by taking into account the anharmonic contribution to the phonon heat capacity. This component of the heat capacity can be calculated using the experimental data of compressibility $K_{\mathrm{T}}$ and thermal expansion coefficient $\alpha\left(C_{\mathrm{p}}-C_{v}=V \alpha^{2} T / K_{\mathrm{T}}\right.$, where $V$ is the molar volume). There are no available data on the compressibility of $\mathrm{BiFeO}_{3}$, accordingly, the anharmonic contribution to the phonon heat capacity is obtained basing on thermal expansion coefficient measured on $\mathrm{Bi}_{1-x} \mathrm{La}_{x} \mathrm{FeO}_{3}$ samples in [9] and the pressure dependence of volume studied for $\mathrm{LaAlO}_{3}$ [10] with a similar structure. Basing on these data, the anharmonic contribution to the phonon heat capacity of $\mathrm{BiFeO}_{3}$ at $300 \mathrm{~K}$ is found to be about $1 \mathrm{~J} / \mathrm{mol} \mathrm{K}$, i.e. less than $1 \%$ of the total heat capacity. The small anharmonic contribution is due to a fairly low thermal expansion coefficient of $\mathrm{BiFeO}_{3}$. The further analysis of the temperature dependence of the phonon heat capacity can be performed whilst the difference between $C_{p}$ and $C_{v}$, can be neglected.

In the most cases, the quantitative analysis of the temperature dependence of heat capacity and separation of the phonon and the anomalous contribution is performed using a simple model, whereby the phonon heat capacity should be governed by the Debye function $C_{v}{ }^{0} \sim D\left(\Theta_{\mathrm{D}} / T\right)$, where $\Theta_{\mathrm{D}}$ is the Debye characteristic temperature. The calculation procedure yields the heat capacity of $\mathrm{BiFeO}_{3}$ with the value $\Theta_{\mathrm{D}} \approx 550 \mathrm{~K}$.

The calculation results of phonon heat capacity of $\mathrm{Bi}_{1-x} \mathrm{Pr}_{x} \mathrm{FeO}_{3}$ using the Debye function are indicated in Figure 1 by the solid line. The $\mathrm{BiFeO}_{3}$ compositions modified with Pr demonstrate the deviations of the experimental points from the calculated phonon heat capacity indicating the existence of the excess heat capacity (Figure 2). The excess component of the heat capacity is found as the difference between the measured $C_{\mathrm{p}}$ and the calculated $C_{\mathrm{p}}{ }^{0}$ phonon heat capacity $\Delta C_{\mathrm{p}}=C_{\mathrm{p}}-C_{\mathrm{p}}{ }^{0}$ (for each composition). The temperature dependence of the anomalous heat capacity $\Delta C_{p}(T)$ is presented 


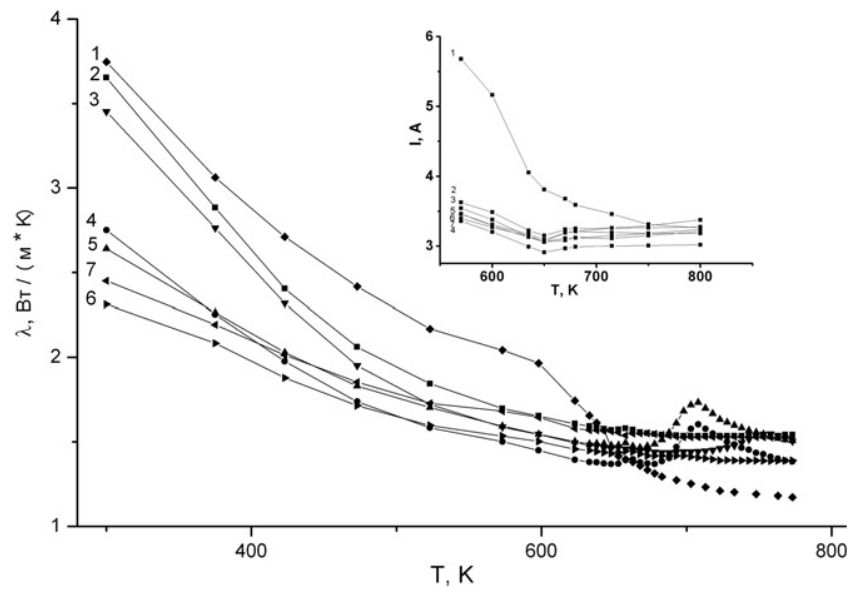

Figure 3. The temperature dependence of thermal conductivity and the mean free path of the phonons $\mathrm{Bi}_{1-x} \mathrm{Pr}_{x} \mathrm{FeO}_{3}$ for $\mathrm{x}=0$ (1), 0.05 (2), 0.10 (3), 0.15 (4), 0.20 (5), 0.40 (6) and 0.50 (7).

in Figure 2. The character of the heat capacity determined by this approach allows for interpreting it as the Schottky anomaly for three-level states separated from the ground state by energy barriers $E_{1}$ and $E_{2}$. They can be atoms of the same type or the atomic group separated by energy barriers $E_{1}$ and $E_{2}$ and having three structurally equivalent positions. In the case of doping with rare-earth elements, a three-level system can emerge due to a distortion of the lattice parameters caused by the polar displacements of bismuth and iron ions from the initial positions, and a change in the bond angle between oxygen octahedra $\mathrm{FeO}_{6}$ [11]. In the general case, the expression for the Schottky heat capacity can be obtained by differentiation of the particle mean energy at the energy levels $C_{p}=\left(k T^{2}\right)^{-1}\left(\left\langle E_{i}^{2}\right\rangle-\left\langle E_{i}\right\rangle^{2}\right)[12]$.

In the case of the three-level model (at arbitrary material mass), the Schottky heat capacity is given as

$$
\Delta C p=\frac{\nu R\left[D_{1}\left(\Delta E_{1} / k T\right)^{2} \exp \left(-\Delta E_{1} / k T\right)+D_{2}\left(\Delta E_{2} k T\right)^{2} \exp \left(-\Delta E_{2} / k T\right)\right]}{\left[1+D_{1} \exp \left(-\Delta E_{1} / k T\right)+D_{2} \exp \left(-\Delta E_{2} / k T\right)\right]^{2}},
$$

where $D_{1}$ and $D_{2}$ are the degeneracy orders of the levels, $R$ is the universal gas constant, and $\nu$ is the number of modes [12]. By comparing the heat capacity calculated by Eq. (1) and that found experimentally, the excess heat capacity $\Delta C_{p}$ was obtained for the following model parameters: $D_{1}=131.66, D_{2}=8.91, E_{1}=0.245 \mathrm{eV}$ and $E_{2}=$ $0.0986 \mathrm{eV}$. There is a sufficiently good agreement between experimental $\Delta C_{\mathrm{p}}(T)$ and calculated temperature dependence of the anomalous heat capacity is (Figure 2). A characteristic $\lambda$-anomaly on $C_{\mathrm{p}}(T)$ curve, caused by the appearance of a magnetic ordering was observed near the antiferromagnetic phase transition $T_{\mathrm{N}}$ (Figures 1 and 2).

For compositions with $\mathrm{x}=0.10,0.15$, and 0.20 , the second anomalies at temperatures $\mathrm{T}=755 \mathrm{~K}$ and $\mathrm{T} \approx 710 \mathrm{~K}$, typical for phase transitions, are observed on the curves of thermal conductivity $v s$ temperature (Figure 3 ), as for the heat capacity (Figure 1) above $\mathrm{T}_{\mathrm{N}}$.

Let us consider the heat transfer mechanism of phonons in the polycrystalline multiferroic $\mathrm{Bi}_{1-\mathrm{x}} \mathrm{Pr}_{\mathrm{x}} \mathrm{FeO}_{3}$. The inset in Figure 3 shows the temperature dependence of the 
phonon mean free path $\mathrm{I}$, which can be derived from the Debye formula $\mathrm{I}=3 \lambda / \mathrm{Cp} \cdot v$ (where $\lambda$ is the thermal conductivity, $\mathrm{Cp}$ is the specific heat, and $v$ is the sound velocity [13]). It can be well seen in the inset in Figure 3, the value of I varies from 3 to 5 angstroms. It follows that the mean free path of phonons is much smaller than the dimensions of the crystallites (which are usually of the order of several microns [9]), so that scattering of phonons at crystallite boundaries can be neglected. Thus, it can be assumed that in $\mathrm{Bi}_{1-\mathrm{x}} \mathrm{Pr}_{\mathrm{X}} \mathrm{FeO}_{3}$, structural distortions (scattering centers) limiting the mean free path of phonons, have the order of magnitude of the lattice constant. According to structural studies on neutron diffraction [11], the distortions in the lattice parameters and changes in the volume of the unit cell are attributable to, rotation of oxygen octahedra centers; the angle between the neighboring octahedra of $\mathrm{FeO}_{6}$ and the polar shifts of bismuth and iron ions from their initial positions increases.

In the temperature region below $\mathrm{T}_{\mathrm{N}}$, the thermal conductivity of the samples increases with decreasing temperature, which is associated with a sharp increase in the mean free path of phonons (see the inset in Figure 3), since the electron-lattice interaction is suppressed by an ordered spin system at the transition to the magnetic phase [14], besides the lattice is compressed [10]. In the temperature region above $T_{N}$ (Figure 3 ), the decrease of thermal conductivity of pure $\mathrm{BiFeO}_{3}$ with heating may be caused by an increase in phonon scattering centers due to lattice distortions [11].

The thermal conductivity of the $\mathrm{Bi}_{1-\mathrm{x}} \mathrm{Pr}_{\mathrm{X}} \mathrm{FeO}_{3}$ system decreasing with increasing concentration $\mathrm{x}$ below $\mathrm{T}_{\mathrm{N}}$ (see Figure 3) is associated with additional local distortions of the crystalline lattice (i.e., phonon scattering centers) which appear due to the substitution of bismuth ions by praseodymium ions of smaller radius.

The slight substitution of praseodymium for bismuth in polycrystalline bismuth ferrite leads to a marked change in the temperature dependences of the heat capacity and thermal conductivity over a wide range of temperatures, as well as to the temperature shift of the antiferromagnetic transition $\mathrm{T}_{\mathrm{N}}$.

\section{Summary}

The results of this study demonstrate that the substitution of praseodymium for bismuth results in a marked shifting of the antiferromagnetic phase transition temperature to higher values. The anomalies observed in the temperature dependences of the heat capacity for the compositions with $x=0.10,0.15$, and 0.20 and their analysis in combination with the structural data suggest their cause to be the structural phase transitions. Doping of bismuth ferrite with rare-earth element Pr, brings an additional contribution to the heat capacity in the temperature range of $240-800 \mathrm{~K}$ that can be interpreted as the Schottky anomaly for three-level states formed as a result of the distortion of the lattice parameters due to the doping.

\section{References}

1. G. A. Smolenskii, and V. M. Yudin, Weak ferromagnetism of some $\mathrm{BiFeO}_{3}-\mathrm{PbFe}_{0.5} \mathrm{Nb}_{0.5} \mathrm{O}_{3}$ perovskites. Sov. Phys. Solid State. 6, 2936 (1965).

2. G. Catalan, and F. Scott, Physics and applications of bismuth ferrite. Adv. Mater. 21 (24), 2463 (2009). DOI: 10.1002/adma.200802849. 
3. J.-H. Lee et al., Variations of ferroelectric off-centering distortion and $3 \mathrm{~d}-4 \mathrm{p}$ orbital mixing in La-doped $\mathrm{BiFeO}_{3}$ multiferroics. Phys. Rev. B. 82, 045113 (2010).

4. D. V. Karpinsky et al., Magnetic and piezoelectric properties of the $\mathrm{Bi}_{1-} x \mathrm{Lax} \mathrm{FeO}_{3}$ system near the transition from the polar to antipolar phase. Phys. Solid State. 56 (4), 701 (2014).

5. D. V. Karpinsky et al., Temperature evolution of the crystal structure of $\mathrm{Bi}_{1}-x \operatorname{Pr} x \mathrm{FeO}_{3}$ solid solutions. Phys. Solid State. 56 (11), 2263 (2014). DOI: 10.1134/S1063783414110146.

6. D. V. Karpinsky et al., Phase coexistence in $\mathrm{Bi}_{1-\mathrm{x}} \mathrm{Pr}_{\mathrm{x}} \mathrm{FeO}_{3}$ ceramics. J. Mater. Sci. 49 (20), 6937 (2014). DOI: 10.1007/s10853-014-8398-6.

7. S. N. Kallaev et al., Heat capacity of multiferroics Bi1-xPrxFeO3. Phys. Solid State . 59 (7), 1477 (2017). DOI: 10.1134/S1063783417070095.

8. J. Zhang, Y.-J. Wu, and X.-J. Chen, Structural evolution and enhanced magnetization of $\mathrm{Bi}_{1}-x \operatorname{Pr} x \mathrm{FeO}_{3}$. J. Magn. Mater. 382, 1 (2015). DOI: 10.1016/j.jmmm.2015.01.061.

9. A. A. Amirov et al., Features of thermal, magnetic, and dielectric properties of the multiferroics BiFeO3 and Bi0.95La0.05FeO3. Phys. Solid State. 51 (6), 1189 (2009). DOI: 10.1134/ S1063783409060183.

10. T. Tohei et al., General rule for displacive phase transitions in perovskite compounds revisited by first principles calculations. Phys. Rev. Lett. 94, 035502 (2005).

11. Arnold, D. C. Knight, K. S. Morrison, and F. D. Lightfoot, Ferroelectric-paraelectric transition in $\mathrm{BiFeO}_{3}$ : crystal structure of the orthorhombic $\beta$ phase. Phys. Rev. Lett. 102, 027602 (2009).

12. R. G. Mitarov et al., Schottky effect in the $\operatorname{Pr}_{3} \mathrm{Te}_{4}-\mathrm{Pr}_{2} \mathrm{Te}_{3}$ system. Phys. Status Solidi A. 30 (2), 457 (1975).

13. E. P. Smirnova et al., Acoustic properties of multiferroic $\mathrm{BiFeO}_{3}$ over the temperature range 4.2-830 K. Eur. Phys. J. B. B83, 39 (2011). DOI: 10.1140/epjb/e2011-20418-1.

14. H. Fujshiro, S. Sugavara, and M. Ikebe, Proceedings of the 10th international conference on phonon scattering in condensed matter. Physica B. 316-317, 331 (2002). DOI: 10.1016/ S0921-4526(02)00500-8.

15. S. N. Kallaev et al., Heat capacity of $\mathrm{BiFeO}_{3}$-based multiferroics. J. Exp. Theor. Phys. 118 (2), 279 (2014). DOI: 10.1134/S1063776114020095. 